\title{
ON TWO QUESTIONS OF HALMOS CONCERNING SUBSPACE LATTICES
}

\author{
W. E. LONGSTAFF AND PETER ROSENTHAL
}

\begin{abstract}
ABSTRACr. An example is constructed of a nonreflexive pentagonal lattice of subspaces. It follows that reflexivity is not invariant under lattice isomorphism, even for finite lattices.
\end{abstract}

Following Halmos [2], [3] we say that a lattice $\mathcal{L}$ of subspaces of a Hilbert space is reflexive if there is a collection of bounded linear operators whose lattice of invariant subspaces is $\mathfrak{L}$. The questions of Halmos [3] referred to in the title are:

(i) Is every subspace lattice which is (isomorphic to) the pentagon reflexive?

(ii) If two finite subspace lattices are isomorphic and one is reflexive, must the other be reflexive?

In this note we exhibit a nonreflexive pentagon, giving a negative answer to the first question. Since Halmos [3] has given an example of a reflexive pentagon, this shows that the second question also has a negative answer.

A nonreflexive pentagon can be described as follows. Let $K$ denote the diagonal operator on $l^{2}$ defined by $K\left\{\alpha_{n}\right\}=\left\{\alpha_{n} / n\right\}$, and define $x=\{1 / n\}$ and $y=\left\{1 / n^{3 / 2}\right\}$. Now let the Hilbert space $\mathcal{H}$ be $l^{2} \oplus l^{2}$ and let $\mathcal{L}$ consist of $\{0\}, l^{2} \oplus\{0\}, \mathcal{G}=\left\{z \oplus K z: z \in l^{2}\right\}, \mathcal{G}_{0}=\left\{z \oplus(K z+\alpha x+\beta y): z \in l^{2}\right.$, $\alpha \in \mathbf{C}, \beta \in \mathbf{C}\}$, and $\mathcal{K}$. We must check that $\mathcal{L}$ is a lattice with Hasse diagram:

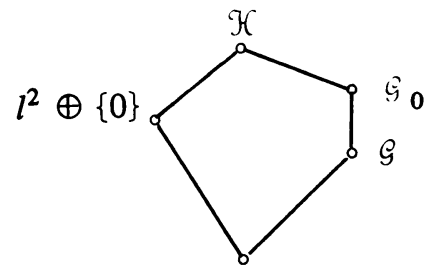

$\{0\}$

All the relations of the above diagram are immediate except that $\mathcal{S}_{0} \cap\left(l^{2}\right.$ $\oplus\{0\})=\{0\}$. But if $K z+\alpha x+\beta y=0$ then $\alpha x+\beta y$ is in the range of $K$. Hence $\sum_{n=1}^{\infty} n^{2}\left|\alpha / n+\beta / n^{3 / 2}\right|^{2}<\infty$, so $\alpha=\beta=0$. Thus $K z=0$, and $z=$ 0 . It follows that $\mathcal{G}_{0} \cap\left(l^{2} \oplus\{0\}\right)=\{0\}$.

Received by the editors September $1,1978$.

AMS (MOS) subject classifications (1970). Primary 47A15, 06A20.

Key words and phrases. Reflexive lattice, invariant subspace. 
THEOREM. The lattice $\mathcal{E}$ is not reflexive.

Proof. Let $\mathbb{Q}$ denote the algebra of all operators leaving the elements of $\mathcal{L}$ invariant. It is apparent (cf. [2], [3]) that $\mathcal{L}$ is reflexive only if every invariant subspace of $\mathcal{Q}$ is an element of $\mathcal{L}$. We show that $\mathscr{T}=\{z \oplus(K z+\lambda y)$ : $z \in l^{2}, \lambda \in \mathbf{C}, K$ and $y$ as above $\}$, which is not an element of $\mathcal{E}$, is invariant under every operator in $\mathbb{Q}$.

Let $T \in Q$. With respect to the decomposition $l^{2} \oplus l^{2}$ of $\mathcal{K}, T$ has the form $T=\left(\begin{array}{l}A B \\ 0\end{array}\right)$. The invariance of $\mathcal{G}$ under $T$ yields $K A+K B K=D K$. For convenience let $C=D-K B$; then $K A=C K$. Since $T$ leaves $\mathcal{G}_{0}$ invariant, $T(0 \oplus y)$ is in $\mathcal{G}_{0}$ and so is $T(0 \oplus y)-(B y \oplus K B y)=0 \oplus C y$. Thus $C y=$ $\alpha x+\beta y$ for some $\alpha$ and $\beta$. Note that $C K=K A$ implies that $C$ leaves the range of $K$ invariant. Hence, by Corollary 2 of [6], $C$ also leaves the range $R$ of $K^{1 / 2}$ invariant. Now $y=K^{1 / 2} x$ is in $\Re$, hence so is $C y$ and so is $\alpha x$. But $x \notin \Re$. Therefore $\alpha=0$ and $C y=\beta y$.

The invariance of $\Re$ under $T$ is now easily seen. For if $z \oplus(K z+\gamma y) \in$ IT, then

$$
\begin{aligned}
\left(\begin{array}{ll}
A & B \\
0 & D
\end{array}\right)\left(\begin{array}{c}
z \\
K z+\gamma y
\end{array}\right) & =\left(\begin{array}{c}
A z+B K z+\gamma B y \\
(C+K B)(K z+\gamma Y)
\end{array}\right) \\
& =\left(\begin{array}{c}
A z+B K z+\gamma B y \\
K A z+\gamma \beta y+K B K z+\gamma K B y
\end{array}\right)=\left(\begin{array}{c}
u \\
K u+\gamma \beta y
\end{array}\right),
\end{aligned}
$$

where $u=A z+B K z+\gamma B y$. Hence $\mathfrak{R}$ is invariant under $T$.

REMARKs. (a) Instead of quoting Corollary 2 of [6] in the above proof, we could use the more general Proposition 5 of [1], once the proof of Proposition 5 is modified; see the remarks following Corollary 2 of [6].

(b) For finite lattices of subspaces of finite-dimensional spaces, the answer to question (ii) above is affirmative. A theorem of Johnson [5] implies that such a lattice is reflexive if and only if it is distributive.

(c) There are many known results concerning reflexive lattices: see [4] for other references to recent work.

\section{REFERENCES}

1. C. Foias, Invariant para-closed subspaces, Indiana Univ. Math. J. 21 (1972), 887-906.

2. P. R. Halmos, Ten problems in Hilbert space, Bull. Amer. Math. Soc. 76 (1970), 887-933.

3. __ Reflexive lattices of subspaces, J. London Math. Soc. 4 (1971), 257-263.

4. K. J. Harrison and W. E. Longstaff, Reflexive subspace lattices in finite-dimensional Hilbert spaces, Indiana Univ. Math. J. 26 (1977), 1019-1025.

5. R. E. Johnson, Distinguished rings of linear transformations, Trans. Amer. Math. Soc. 111 (1964), 400-412.

6. E. Nordgren, M. Radjabalipour, H. Radjavi and P. Rosenthal, On invariant operator ranges, Trans. Amer. Math. Soc. (to appear).

Department of Mathematics, University of Western Australia, Perth, Australla

Department of Mathematics, University of Toronto, Toronto, Ontario, Canada M5S 1A7 